\title{
Neutrophil elastase ameliorates matrix metalloproteinase-9 to promote lipopolysaccharide-induced acute lung injury in mice ${ }^{1}$
}

\author{
Yingfei Guo ${ }^{\mathrm{I}, \mathrm{II}}$, Lingyun Ma ${ }^{\mathrm{I}, \mathrm{II}}$, Fei Zhang ${ }^{\mathrm{I}}$, Rongju Sun ${ }^{\mathrm{I}}$, Tanshi Li \\ DOI: http://dx.doi.org/10.1590/S0102-865020160060000004 \\ IDepartment of Emergency, General Hospital of PLA, Beijing, China. Design the protocol, acquisition and analysis of data, technical procedures, \\ manuscript writing, final approved. \\ IIDepartment of Emergency or Respiration, First Affiliated, General Hospital of PLA, Beijing, China. Statistical analysis, technical procedures, final \\ approved the manuscript.
}

\begin{abstract}
PURPOSE: To investigate the regulatory roles of neutrophil elastase(NE) and matrix metalloproteinase-9(MMP-9) in lipopolysaccharide (LPS)-induced acute lung injury (ALI) in mice.

METHODS: To construct LPS-induced ALI mouse models, wild-type C57BL/6 mice were administered $5.0 \mathrm{mg} / \mathrm{kg}$ of LPS through endotracheal, and/or $1.0 \mathrm{mg} / \mathrm{kg}$ of ONO-5046, and/or $20.0 \mathrm{mg} / \mathrm{kg}$ of chemically modified tetracycline-3 (CMT-3) by gavage. The levels of MMP-9, tissue inhibitor of metalloprotease-1, interleukin (IL)-6 were detected by real time RT-PCR at 6 h, 24 h and 48 h, and tumor necrosis factor (TNF), lung wet-dry weight ratio, white blood cell (WBC) count and polymorphonuclear (PMN) count in bronchoalveolar lavage fluid (BALF) were tested at $48 \mathrm{~h}$ after administration. The 5-day survival analysis of the ALI mice was also performed.
\end{abstract}

RESULTS: Both ONO-5046 and CMT-3, regardless of being used individually or combined, significantly reduced the levels of MMP9, IL-6, and TNF in lung tissue as well as in BALF, and the WBC and PMN count in BALF. Combined treatment with ONO-5046 and CMT-3 remarkably improved the survival rate of ALI mice.

CONCLUSION: Neutrophil elastase synergizes with matrix metalloproteinase-9 to promote and regulate the release of inflammatory mediators and the infiltration of inflammatory cells, consequently affecting the survival of lipopolysaccharide-induced acute lung injury mice.

Key words: Leukocyte Elastase. Matrix Metalloproteinases. Lipopolysaccharides. Acute Lung Injury. Mice. 


\section{Introduction}

The pathophysiological characteristics of acute lung injury (ALI) and acute respiratory distress syndrome (ARDS) include direct or indirect injury to the alveolar epithelial and capillary endothelial cells, increased permeability of the alveolar-capillary membrane, infiltration of inflammatory cells, and release of inflammatory mediators, which in turn lead to interstitial and alveolar pulmonary edema, oxygenation damage, progressive hypoxemia, and dyspnea. Owing to the severe clinical manifestations, ALI and ARDS have considerably high mortality rates. ALI and ARDS can be induced by a variety of conditions, including sepsis, pancreatitis, ischemia-reperfusion injury, and inhalation injury ${ }^{1-4}$. However, the exact mechanisms underlying the onset of ALI and ARDS remain unclear.

Matrix metalloproteinases (MMPs) are zinc-dependent endopeptidases that belong to a large family of proteases known as the metzincin superfamily. MMPs are involved in a variety of cellular activities, including the proteolysis of cell surface receptors, release of apoptosis-inducing ligands, and cytokine activation, as well as cell proliferation, migration, adhesion, differentiation, and apoptosis. MMPs play critical roles in tissue remodeling processes, such as neovascularization, tissue reconstruction, cirrhosis, arthritis, metastasis, and in the formation of aortic aneurysm.

MMPs are closely related to the pathogenesis of lung injury. Among the different types of lung injuries, ALI is known to be closely related to the production of inflammatory mediators from neutrophils and the release of cytotoxic molecules. In particular, the accumulation of neutrophils in the microvasculature of the lung is considered critical to the pathogenesis of ALI MMP-9 is a key factor that regulates neutrophil migration and activation. MMP-9 inhibition by MMP inhibitors can reduce neutrophil transmigration. The elevated level of MMP-9 in the bronchoalveolar lavage fluid (BALF) in ALI has been suggested to play a role in neutrophil-mediated ALI and the corresponding inflammatory responses ${ }^{5-9}$.

Neutrophil elastase (NE) is a protease involved in the processes of tissue remodeling and inflammation that characterize a number of diseases, including hereditary emphysema, chronic obstructive pulmonary disease (COPD), cystic fibrosis (CF), ARDS, ischemic-reperfusion injury, and rheumatoid arthritis. Human NE is a well-characterized serine protease that has been shown to serve as a biomarker of pulmonary inflammation in both $\alpha-1$ antitrypsin deficiency and CF. The dysregulation or inhibition of NE could repress the pulmonary inflammatory responses in $\mathrm{CF}$ and COPD. NE contributes to the pathogenesis of as well as the repair process following ALI and ARDS. In COPD, NE is known to activate MMPs, including MMP-9. However, the exact role of NE in ALI and ARDS is unclear ${ }^{10,11}$.

In this study, we examined the levels of MMP-9, NE, and inflammatory mediators in the lung tissues and BALF from LPS-induced ALI mice to investigate the role of MMP-9 and the involvement of NE in this pathogenic process.

\section{Methods}

All animal experiments had been authorized by the Animal Care and Use Ethics Committee of our institution and approved by the local Animal Protection Committee. The experiments were performed according to the Guide for the Care and Use of Laboratory Animals.

\section{Reagents}

Lipopolysaccharide (E.Coli 055:B5) was from Sigma (L2880, CA, USA); Sivelestat sodium, ONO-5046 was from Sanctity Co (CAS: 127373-66-4, Harbin, China); Metastat, CMT3 was from CollaGenex Pharmaceuticals (CAS: 305838-77-1, PA, USA).

\section{Experimental design, groups and LPS-induced ALI mouse model}

The assay of NE promoting MMP9, TIMP induced by LPS was designed to LPS and LPS+NEI groups that 15 mice were in each group. Lung tissue samples were obtained at each time point of $6 \mathrm{~h}, 24 \mathrm{~h}$ or $48 \mathrm{~h}$, and bronchoalveolar lavage fluid (BALF) were collected at times of $48 \mathrm{~h}$ after administration, namely the longest time for $48 \mathrm{~h}$ after LPS or NEI treatment. MMP9, TIMP and IL-6 levels in lung tissues were determined at the indicated time points of $6 \mathrm{~h}, 24 \mathrm{~h}$ or $48 \mathrm{~h}$ respectively. The assay of 5-day survival rate was divided into 5 groups of sham, LPS, LPS+NEI, LPS+CMT, LPS+NEI+CMT. Each group includes 15 mice. The 5 -day survival in each group was continuously observed and calculated.

The NEI and CMT collaborative experiments were divided into 4 groups of control (CON), NEI, CMT and NEI+CMT groups that 15 mice were in each group. Lung wet-dry ratio, leukocyte and neutrophil cell count, IL-6, and TNF levels in BALF were detected at time of $48 \mathrm{~h}$ after administration. 
Wild-type C57BL/6 mice were intratracheally administered $5.0 \mathrm{mg} / \mathrm{kg}$ of LPS. Control mice were injected with $1 \mathrm{ml}$ of vehicle (sterile saline) by gavage as a sham treatment. Mice in the MMP inhibitor (MMP) group were administered 20.0 $\mathrm{mg} / \mathrm{kg}$ of chemically modified tetracycline-3 (CMT-3) in $1.0 \mathrm{ml}$ of vehicle consisting of N-Methylpyrrolidone, propylparaben, methylparaben, ethanol, and $2 \%$ carboxymethyl cellulose, by gavage. Mice in the NE inhibitor (NEI) group were administered $1.0 \mathrm{mg} / \mathrm{kg}$ of ONO-5046 in $1.0 \mathrm{ml}$ of vehicle containing $0.02 \%$ of dimethyl sulfoxide. ALI was confirmed on the basis of manifestations such as alveolar capillary congestion, hemorrhage, neutrophil infiltration, and increased thickness of the alveolar wall, as described in a previous study ${ }^{12}$.

\section{Tissue preparation and measurement of the lung dry/wet weight ratio}

A thoracotomy was performed, and the heart and lungs were excised from the mice. The left lung was separated and weighed after ligation of the left main bronchus. The lung was then dried in a drying oven until a constant weight was obtained, and the lung dry/wet weight ratio was then calculated.

\section{$B A L F$ analysis}

Bronchoalveolar lavage (BAL) was performed using 0.3-ml aliquots of sterile phosphate buffered saline (PBS). After centrifugation, the cells were separated from the supernatant fraction. The BALF samples were then frozen at $-80^{\circ} \mathrm{C}$. Red blood cells were eliminated from the cell fraction by hypotonic lysis, and the cell-free supernatant was harvested. Each lung was lavaged with three aliquots of sterile saline. The total and differential white blood cell (WBC) counts of the BAL cells were performed. The lung was then removed and its water content was measured on the basis of the lung dry/wet weight ratio. A blood sample was obtained by cardiac puncture.

The cell-free BALF samples harvested from the mice in each group, namely, control (sham), LPS, LPS + NEI, LPS + CMT, LPS + NEI + CMT, control + NEI, control + CMT, and control + NEI + CMT, were analyzed in triplicate for WBC count, polymorphonuclear (PMN) count, and interleukin (IL)-6 and tumor necrosis factor (TNF) levels, using commercially available ELISA kits. The results were expressed as $\mathrm{pg} / \mathrm{BAL}$ after correcting for the volume of BALF that was recovered from each mouse $(\geq 3.0 \mathrm{ml}$ of BALF was recovered from a total of $4 \mathrm{ml}$ PBS administered to each mouse).

\section{Real-time polymerase chain reaction}

Mice were sacrificed and dissected immediately after a lethal dose of anesthesia. Total RNA was isolated from their lungs. For each reaction, $0.5 \mu \mathrm{g}$ of RNA was reverse transcribed using the Omniscript reverse transcriptase kit (Qiagen, Inc., Valencia, CA, USA) according to the manufacturer's protocol. Primers for the specific detection of MMP-9, IL-6, and tissue inhibitor of metalloproteinase-1 (TIMP-1) were designed using the Real-Time Polymerase Chain Reaction (PCR) software (Integrated DNA Technologies, Inc., Coralville, IA, USA). The sequences of these primers were as follows: MMP-9 (NM013599): 3'-GATCCCCAGAGCGTCATTC-5', 3'-CCACCTTGTTCACCTCATTTTG-5'; (NM011593): 3'-CTCAAAGACCTATAGTGCTGGC-5', 3'-CAAAGTGACGCTCTGGTAG-5'; IL-6 (NM031168): 5' - AT G A A G T T C C T C T C T GCA A G A GA C T - 3', 5 ' - C A C TA G G T T T G C C GA G T A G AT C T C - 3'; $\beta$-actin: 5'-TGGAATCCTGTGGCATCCATGAA-3', 5'-TAAAACGCAGCTCAGTAACAGTC-3'. The thermal cycling conditions entailed the following steps: 1) polymerase activation at $95^{\circ} \mathrm{C}$ for $\left.3 \mathrm{~min} ; 2\right) 45$ cycles of denaturation at $95^{\circ} \mathrm{C}$ for 10 $\mathrm{s}$ followed by annealing and extension at $60^{\circ} \mathrm{C}$ for $30 \mathrm{~s}$; and 3) generation of a DNA melting curve for determining amplicon specificity. All experiments were performed in duplicate. For each PCR reaction, the sample was amplified in triplicate on an Applied Biosystems 7500 Real-Time PCR System (Applied Biosystems, Foster City, CA, USA). $\beta$-Actin was used as the internal control. The relative levels of each examined gene were calculated according to the manufacturer's instructions.

\section{Survival analysis}

Survival analysis was performed on the mice in each group over a period of $5 \mathrm{~d}(120 \mathrm{~h})$, and the survival rate was calculated accordingly. Data were expressed as mean \pm SEM. Intergroup differences were evaluated by one-way ANOVA using LPS or saline treatment as a factor. $P<0.05$ was considered statistically significant.

\section{Results}

\section{MMP-9 and TIMP-1 expression and ratio of MMP- 9/TIMP-1}

An ALI mouse model was established by intratracheal administration of LPS in C57BL/6 mice. Lung tissue samples 
were obtained from these mice with a lethal dose of anesthesia at the indicated times of $6 \mathrm{~h}, 24 \mathrm{~h}$, or $48 \mathrm{~h}$ after administration of LPS and/or NEI. Total RNA was extracted from these samples and used for the detection of MMP-9, IL-6, and TIMP-1 mRNA expression and for the determination of the MMP-9/TIMP-1 ratio in the lung tissues in ALI mice. Our results showed that the expression level of MMP-9 was significantly elevated in the lung tissues obtained from LPS-induced ALI mice compared to that in the lung tissues obtained from control mice, in a time-dependent manner. Moreover, the expression of the natural MMP-9 inhibitor TIMP-1 and the ratio of MMP-9/TIMP-1 in the LPS-treated group of mice were significantly higher than those in the sham group (Figure 1).
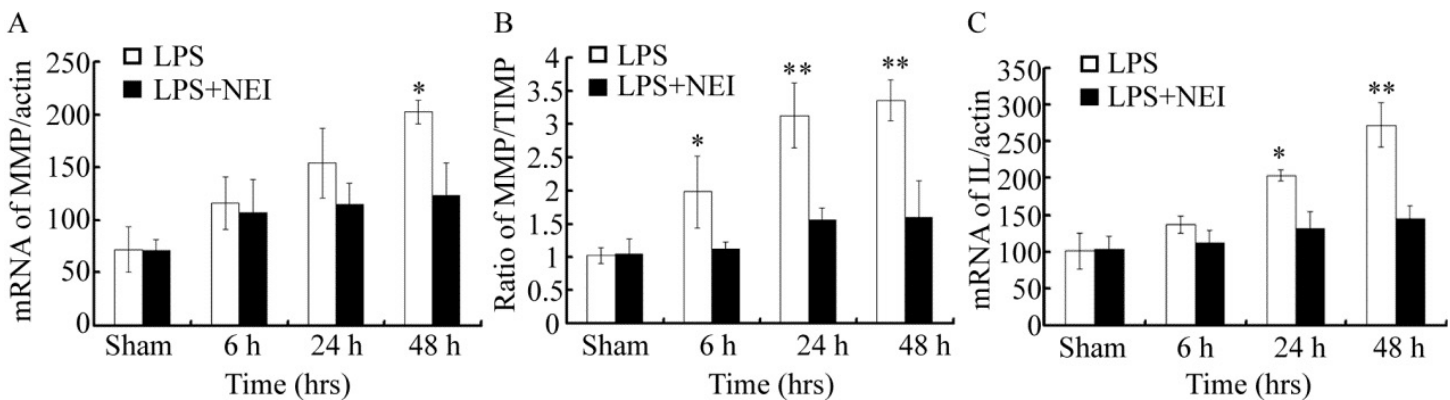

FIGURE 1 - NE regulates MMP-9, TIMP-1 and IL-6 expression in LPS-induced ALI mice. C57BL6 mice were treated with $5.0 \mathrm{mg}$ of LPS for 0 h, 6 $\mathrm{h}, 24 \mathrm{~h}$ and $48 \mathrm{~h}$ or saline by IT. mRNA levels of MMP-9 (A), the ratio of MMP-9/TIMP-1 (B) and IL-6 mRNA (C) in lung tissues from ALI mice or control mice were determined by real time RT-PCR. In LPS-induced ALI mice, the expression of MMP-9 and IL-6 and the ratio of MMP-9/TIMP-1 were elevated in a time-dependent manner. NEI treatment significantly inhibited the increase of MMP-9 and IL-6 mRNA levels induced LPS treatment. Similar change was observed for the ratio of MMP-9/TIMP-1. Data was expressed as mean \pm SEM; $n=15$ for con $(0 \mathrm{~h})$ and ALI $6 \mathrm{~h}, 24 \mathrm{~h}$ and $48 \mathrm{~h}$ groups. Single Asterisk indicates $\mathrm{p}<0.05$; double asterisk indicates $\mathrm{p}<0.01$; triple asterisk indicates $\mathrm{p}<0.001$.

\section{Regulation of expression of MMP-9, TIMP-1, and IL-6}

Lung tissue samples were obtained from mice in the LPS and LPS + NEI groups at the time points of $6 \mathrm{~h}, 24 \mathrm{~h}$ and $48 \mathrm{~h}$ after administration of LPS and/or NEI. The expression of NE, MMP-9, TIMP-1, and IL-6 mRNA and the ratio of MMP-9/TIMP-1 in these samples were examined. The results showed that the expression of NE, MMP-9, and IL-6 was significantly downregulated in a timedependent manner in the LPS + NEI group as compared to that in the LPS group. In the control group, NEI treatment did not show any significant difference in TIMP-1 expression (Figure 1).

\section{Role of NE and MMP-9 in pulmonary edema, neutrophil infiltration, and regulation of expression of inflammatory mediators}

The left lungs from mice in the LPS, LPS + NEI, and LPS + CMT groups were excised after a lethal dose of anesthesia, and BALF was obtained for calculation of the lung dry/wet weight ratio and for WBC count and PMN count at time of $48 \mathrm{~h}$ after administration of LPS and/or NEI. The results showed that the lung dry/wet weight ratio in the LPS + NEI and LPS + CMT groups was significantly lower than that in the LPS group. Moreover, the WBC count, PMN count, and the expression of inflammatory mediators IL-6 and TNF in the BALF from the LPS + NEI and LPS + CMT groups were significantly lower than those in the BALF from the LPS group (Figures 2 and 3).
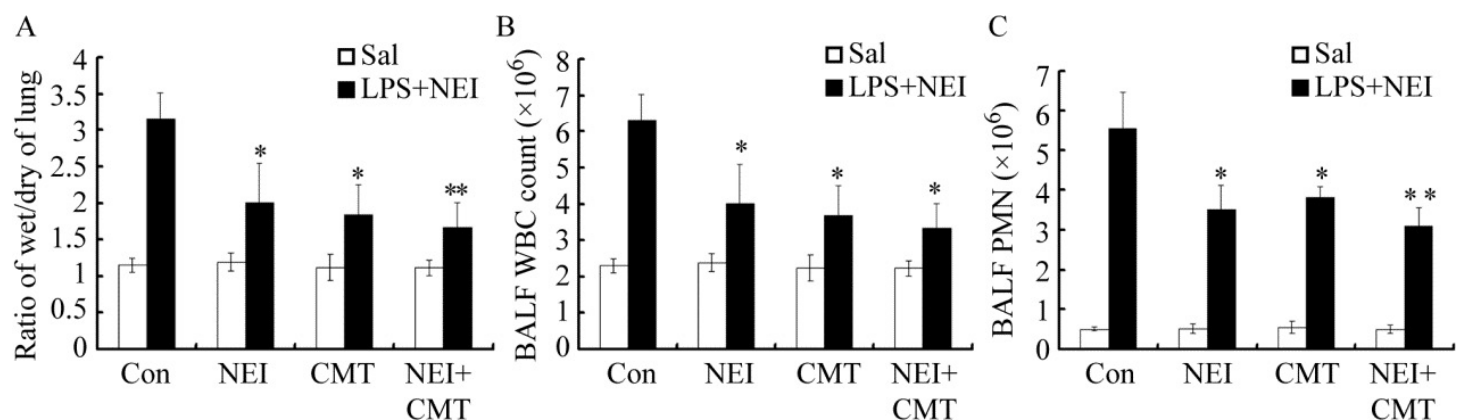

FIGURE 2 - NE and MMP contribute to acute lung edema and PMN infiltration in LPS-induced ALI mice. A. Lung dry/wet weight ratio was measured in LPS-induced ALI mice and LPS-induced ALI mice treated with NEI and/or CMT. WBC (B) and PMN (C) counts in BALF samples were quantified $48 \mathrm{~h}$ after NEI and/or CMT treatment in LPS-induced ALI mice. WBC and PMN in BALF decreased after NEI and/or CMT treatment. Data were expressed as mean $\pm \operatorname{SEM}(\mathrm{n}=15)$. Asterisk indicates $\mathrm{p}<0.05$. Double asterisk indicated $\mathrm{p}<0.01$, compared to the control mice. 

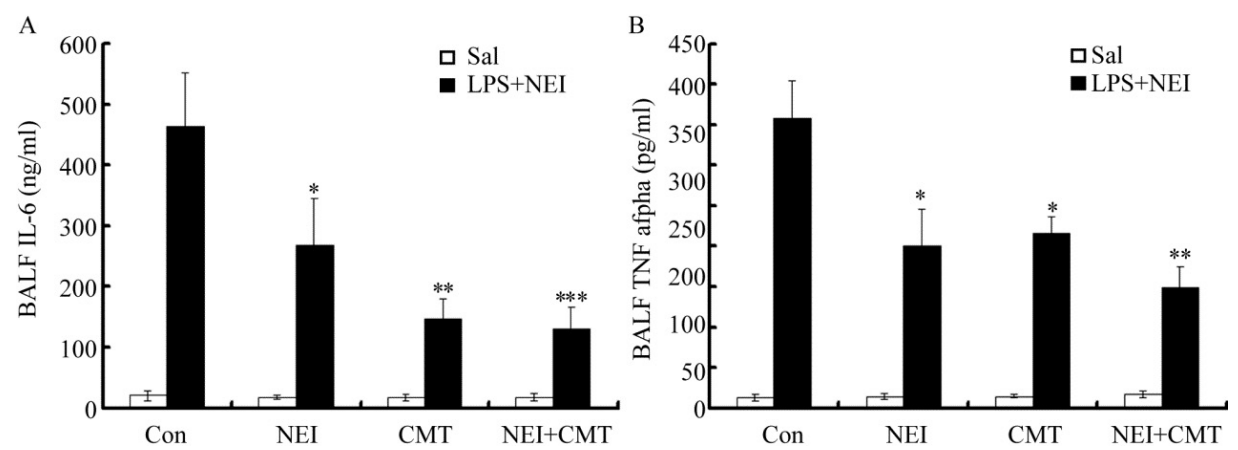

FIGURE 3 - MMP and NE participate in inflammatory responses in lung BALF of LPS-induced ALI mice. C57BL6 mice were treated with 5.0 $\mathrm{mg} / \mathrm{kg}$ LPS (LPS, n=15), $5.0 \mathrm{mg} / \mathrm{kg}$ LPS + $2.0 \mathrm{mg} / \mathrm{kg}$ ONO5046 (LPS+NEI, $\mathrm{n}=15), 5.0 \mathrm{mg} / \mathrm{kg} \mathrm{LPS} \mathrm{+} 2.0 \mathrm{mg} / \mathrm{kg}$ CMT-3 (LPS + CMT), LPS +NEI + CMT or alone saline (sham, $n=15$ ) by IT. IL- 6 and TNF levels in BALF of LPS-induced ALI mice were measured by ELISA. Panel A showed that NEI treatment and CMT treatment, individually or combined, significantly reduced the IL-6 level compared to the control. Panel B showed that NEI treatment and CMT treatment, individually or combined, significantly reduced the TNF level compared to the control. $*$ indicates $\mathrm{p}<0.05$ ( $\mathrm{n}=15$ ) compared to the control. $* *$ indicates $\mathrm{p}<0.01$ compared to the control. $* * *$ indicate $\mathrm{p}<0.001$ compared to the control.

\section{Effect of NE and MMP-9 on survival rate}

Survival analysis was performed on all 75 mice over a period of 5 days $(120 \mathrm{~h})$. The results showed that combined treatment with NEI and CMT, rather than treatment with NEI or CMT alone, significantly improved the survival rate of ALI mice (Figure 4).

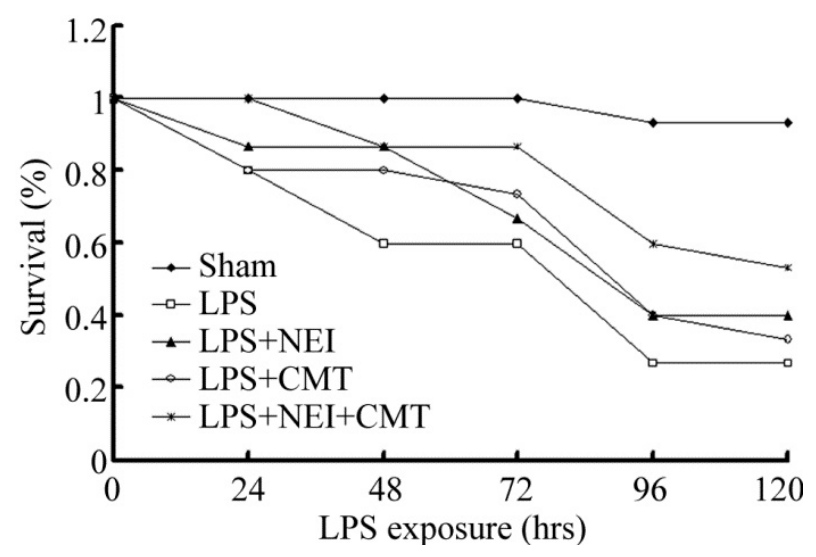

FIGURE 4 - Survival analysis indicates the effect of NE and/or MMP-9 on the survival rate of LPS-induced ALI mice. Survival rate was evaluated in LPS, LPS+NEI, LPS+CMT and LPS+NEI+CMT. Within 5 days, the survival rate of the LPS $+\mathrm{NEI}+\mathrm{CMT}$ group was effectively improved compared to the control. The difference was significant $(\mathrm{p}<0.05, \mathrm{n}=15)$.

\section{Discussion}

Previous studies have shown that neutrophil infiltration and inflammatory mediator release play important roles in ALI induced by injury to the pulmonary microcirculation and alveolar- capillary barrier. The changes in the expression levels of MMP9 and NE in the LPS-induced ALI mouse models used in this study were in agreement with this observation. We observed that the expression of MMP-9 and TIMP-1 and the ratio of MMP-9/ TIMP-1 in the lung tissues of mice with LPS-induced ALI were significantly higher than those in the control mice, and that the differences in these levels were dependent on the duration of LPS treatment. Moreover, the WBC and PMN counts and the levels of IL-6 and TNF in the BALF from ALI mice were significantly higher than those in the BALF from control mice. These results suggested that MMP-9 and NE might promote the activation and accumulation of inflammatory cells in the interstitial lung tissue and alveolar-capillary barrier ${ }^{1-3,11-17}$.

We also observed that treatment with ONO-5046, a small molecule inhibitor of NE, remarkably inhibited the expression of NE and MMP-9 in a time-dependent manner, without significantly affecting TIMP-1 expression, thereby significantly altering the MMP-9/TIMP-1 ratio $^{18-20}$. NEI treatment also inhibited the expression of IL-6 in a time-dependent manner. These results suggested that NE might regulate MMP-9 expression in LPSinduced ALI, thereby regulating the ratio of MMP-9/TIMP-1 and the expression of inflammatory mediators. Inhibition of NE in LPS-induced ALI also reduced the levels of IL-6 and TNF in the BALF, suggesting that NE might not only regulate the expression of inflammatory mediators but also affect their release.

Treatment with the MMP-9 inhibitor CMT-3 also decreased the expression levels of MMP-9, IL-6, and TNF and the MMP-9/TIMP-1 ratio. This could promote the release of IL-6 and TNF in LPS-induced ALI, which in turn could be inhibited by TIMP-1. Therefore, our findings suggested that the level of 
MMP-9, in particular, the ratio of MMP-9/TIMP-1, could play an important role in the expression and release of inflammatory mediators.

The results of BALF analysis showed that treatment with both NEI and CMT significantly reduced the WBC and PMN counts, indicating that both NE and MMP-9 could regulate the activation and accumulation of inflammatory cells in LPS-induced ALI. In addition, treatment with both NEI and CMT significantly reduced the weight of lungs with ALI, which indicated the role of NE and MMP-9 in the development of pulmonary edema in ALI.

Survival analysis showed that combined treatment with NEI and CMT significantly improved the survival rate of ALI mice.

\section{Conclusions}

Lipopolysaccharide treatment induced the expression of neutrophil elastase and matrix metalloproteinase-9, and the activation and accumulation of inflammatory cells in acute lung injury mouse models. NE may regulate the expression of MMP-9 and the ratio of MMP-9/TIMP-1, thereby regulating the expression and release of inflammatory mediators, leading to pulmonary edema and lung injury, and consequently affecting the survival rate of ALI mice.

\section{References}

1. Grommes J, Soehnlein O. Contribution of neutrophils to acute lung injury. Mol Med. 2011 Mar-Apr;17(3-4):293-307. doi: 10.2119/ molmed.2010.00138.

2. Ware LB, Matthay MA. The acute respiratory distress syndrome. $\mathrm{N}$ Engl J Med. 2000 May 4;342(18):1334-49. doi: 10.1056/ NEJM200005043421806.

3. Rubenfeld GD, Caldwell E, Peabody E, Weaver J, Martin DP, Neff M, Stern EJ, Hudson LD. Incidence and outcomes of acute lung injury. N Engl J Med. 2005 Oct 20;353(16):1685-93. doi: 10.1056/ NEJMoa050333.

4. Goss CH, Brower RG, Hudson LD, Rubenfeld GD; ARDS Network. Incidence of acute lung injury in the United States. Crit Care Med. 2003 Jun;31(6):1607-11. doi: 10.1097/01. CCM.0000063475.65751.1D.

5. Ware LB. Pathophysiology of acute lung injury and the acute respiratory distress syndrome. Semin Respir Crit Care Med. 2006 Aug;27(4):337-49. doi: 10.1055/s-2006-948288.

6. Chignard M, Balloy V. Neutrophil recruitment and increased permeability during acute lung injury induced by lipopolysaccharide. Am J Physiol Lung Cell Mol Physiol. 2000 Dec;279(6):L1083-90. PMID: 11076798
7. Fung YL, Silliman CC. The role of neutrophils in the pathogenesis of transfusion-related acute ling injury. Transfus Med Rev. 2009 Oct;23(4):266-83. doi: 10.1016/j.tmrv.2009.06.001.

8. Delclaux C, Delacourt C, D'Ortho MP, Boyer V, Lafuma C, Harf A. Role of gelatinase B and elastase in human polymorphonuclear neutrophil migration across basement membrane. Am J Respir Cell Mol Biol. 1996 Mar;14(3):288-95. doi: 10.1165/ ajrcmb.14.3.8845180.

9. Keck T, Balcom JH 4th, Fernández-del Castillo C, Antoniu BA, Warshaw AL. Matrix metalloproteinase-9 promotes neutrophil migration and alveolar capillary leakage in pancreatitis-associated lung injury in the rat. Gastroenterology. 2002 Jan;122(1):188-201. doi: 10.1053/gast.2002.30348.

10. Roghanian A, Sallenave JM. Neutrophil elastase (NE) and NE inhibitors: canonical and noncanonical functions in lung chronic inflammatory diseases (cystic fibrosis and chronic obstructive pulmonary disease). J Aerosol Med Pulm Drug Deliv. 2008 Mar;21(1):125-44. doi: 10.1089/jamp.2007.0653.

11. Kim JH, Suk MH, Yoon DW, Lee SH, Hur GY, Jung KH, Jeong HC, Lee SY, Lee SY, Suh IB, Shin C, Shim JJ, In KH, Yoo SH, Kang $\mathrm{KH}$. Inhibition of matrix metalloproteinase- 9 prevents neutrophilic inflammation in ventilator-induced lung injury. Am J Physiol Lung Cell Mol Physiol. 2006 Oct;291(4):L580-7. doi: 10.1152/ ajplung.00270.2005

12. Imanaka H, Shimaoka M, Matsuura N, Nishimura M, Ohta N, Kiyono H. Ventilator-induced lung injury is associated with neutrophil infiltration, macrophage activation, and TGF-beta 1 mRNA upregulation in rat lungs. Anesth Analg. $2001 \mathrm{Feb} ; 92(2)$ :42836. doi: 10.1213/00000539-200102000-00029.

13. Tremblay GM, Janelle MF, Bourbonnais Y. Anti-inflammatory activity of neutrophil elastase inhibitors. Curr Opin Investig Drugs. 2003 May;4(5):556-65. PMID: 12833649.

14. Stoller JK, Aboussouan LS. Alpha1-antitrypsin deficiency. Lancet. 2005 Jun 25-Jul 1;365(9478):2225-36. doi: 10.1016/S01406736(05)66781-5.

15. Yang T, Zhang J, Sun L, Zhu X, Li J, Wang J, Chen H, Bao R, Deng X, Hou J, Liu Y. Combined effects of a neutrophil elastase inhibitor (sivelestat sodium) and a free radical scavenger (edaravone) on lipopolysaccharide-induced acute lung injury in rats. Inflamm Res. 2012 Jun;61(6):563-9. doi: 10.1007/s00011-012-0445-7.

16. Siedle B, Hrenn A, Merfort I. Natural compounds as inhibitors of human neutrophil elastase. Planta Med. 2007 May;73(5):401-20. doi: 10.1055/s-2007-967183.

17. Fujino N, Kubo H, Suzuki T, He M, Suzuki T, Yamada M, Takahashi T, Ota C, Yamaya M. Administration of a specific inhibitor of neutrophil elastase attenuates pulmonary fibrosis after acute lung injury in mice. Exp Lung Res. 2012 Feb;38(1):28-36. doi: 10.3109/01902148.2011.633306

18. Ferry G, Lonchampt M, Pennel L, de Nanteuil G, Canet E, Tucker GC. Activation of MMP-9 by neutrophil elastase in an in vivo model of acute lung injury. FEBS Lett. 1997 Feb 3;402(2-3):111-5. doi: 10.1016/S0014-5793(96)01508-6.

19. Itoh Y, Nagase H. Preferential inactivation of tissue inhibitor of metalloproteinases-1 that is bound to the precursor of matrix metalloproteinase 9 (progelatinase $\mathrm{B}$ ) by human neutrophil elastase. J Biol Chem. 1995 Jul 14;270(28):16518-21. doi: 10.1074/ jbc.270.28.16518 
Guo Y et al.

20. Tao W, Miao QB, Zhu YB, Shu YS. Inhaled neutrophil elastase inhibitor reduces oleic acid-induced acute lung injury in rats. Pulm Pharmacol Ther. 2012 Feb;25(1):99-103. doi: 10.1016/j. pupt.2011.12.006.

\section{Correspondence:}

\section{Rongju Sun}

Department of Emergency, General Hospital of PLA

28 Fuxing Road, Beijing 100853 China

Phone: +861066938473

rongjusun@163.com

Received: Feb 24, 2016

Review: Apr 22, 2016

Accepted: May 23, 2016

Conflict of interest: none

Financial source: National Natural Science project of China ( $\left.\mathrm{N}^{\circ} 81272088\right)$

${ }^{1}$ Research performed at Department of Emergency, General Hospital of PLA, Beijing, China. 